\title{
Forecasting value-at-risk by encompassing CAViaR models via information criteria
}

\author{
Sangyeol Lee ${ }^{1} \cdot J_{\text {ungsik } N^{2}}^{2}$ \\ ${ }^{12}$ Department of Statistics, Seoul National University \\ Received 25 September 2013, revised 26 October 2013, accepted 1 November 2013
}

\begin{abstract}
This paper proposes a new method of VaR forecasting using the conditional autoregressive VaR (CAViaR) models and information criteria. Instead of using a single CAViaR model, we propose to utilize several candidate CAViaR models during a forecasting period. By adopting the Akaike and Bayesian information criteria for quantile regression, we can update not only parameter estimates but also the CAViaR specifications. We also propose extended CAViaR models with a constant location parameter. An empirical study is provided to examine the performance of the proposed method. The results suggest that our method shows more stable performance than those using a single specification.
\end{abstract}

Keywords: CAViaR models, GARCH models, information criteria, quantile regression, value-at-risk.

\section{Introduction}

Various risk measures are key components for modern financial risk management. Among others, value-at-risk (VaR) has been extensively studied for several decades and expected shortfall also has attracted attention from researchers in recent years. The VaR measures the maximum potential loss of a given portfolio over a prescribed period, so it is determined by the unknown distribution of loss, or equivalently, the distribution of the return during the period. Hence, the VaR is a particular quantile of future portfolio values or returns.

In the VaR literature, researchers have mainly focused on how to forecast the VaR and how to assess the quality of given forecasts. The former may be concerned by financial institutions and the latter by their regulators. With regard to the VaR prediction, many competing methods have been proposed. For a review of those methods, we refer to Kuester et al. (2006). As examples of VaR analysis for Korean time series, Shim and Hwang (2011) studied the conditional autoregressive VaR (CAViaR) models by Engle and Manganelli (2004) based on support vector quantile regression; Kim and Lee (2011) considered the VaR forecasting of portfolios using conditional copula; Lee and Noh (2010) studied the forecasting based on

$\dagger$ This work was supported by the National Research Foundation of Korea (NRF) grant funded by the Korea government (MSIP) (No.2012R1A2A2A01046092).

1 Professor, Department of Statistics, Seoul National University, Seoul 151-747, Korea

2 Corresponding author: Post doctor, Department of Statistics, Seoul National University, Seoul 151-747, Korea. E-mail: nohjssunny@gmail.com 
quantile regression for GARCH models; Choi et al. (2007) applied the multivariate GARCH models. Among the various forecasting methods, this paper focuses on the CAViaR models which has attracted a great deal of attention recently.

Engle and Manganelli (2004) proposed to model the VaR directly rather than the whole distribution and to estimate the single quantile via quantile regression method. They presented a class of CAViaR models for the parametric specification of the VaR. Many studies suggest that the CAViaR method shows satisfactory performance in various situation: see, for instance, Kuester et al. (2006) and Bao et al. (2006). Since the CAViaR models consist of various specifications, one may encounter a practical problem of choosing a proper specification among many of them. In this paper, we address this model selection issue and propose a new method of VaR forecasting where we encompass several CAViaR models via information criteria. It is noteworthy that only a few papers considered the selection of the VaR model. Gargallo et al. (2010) considered a Bayesian method of VaR forecasting and proposed a selection criterion for VaR models based on the Bayes factor.

Since the CAViaR method is based on the quantile regression, one simple way to select a CAViaR model is to utilize the Akaike and Bayesian information criteria (AIC and BIC) for the quantile regression. Further, we propose to use multiple CAViaR specifications instead of using only a single specification during a forecasting period. That is, at each time, we may update not only parameter estimates but also the CAViaR model specifications based on the AIC or BIC. This method takes into account the possibility that the CAViaR specification may change over time. To examine the performance of our method, we implement one-dayahead VaR forecasting for real data of daily returns. The empirical results below illustrate that the performance of the method is reasonable and more stable than any single CAViaR model.

In what follows, we introduce the notation and definition of the VaR used in this paper. Let $\left\{P_{t}\right\}$ be daily prices of a portfolio. In most cases, the price processes are non-stationary and their $\log$ returns form stationary processes. Denote by $Y_{t}=100 \cdot \log P_{t} / P_{t-1}$ the daily $\log$ return in percentage. Let $\mathcal{F}_{t}$ denote the information set up to time $t$. The conditional daily VaR at $100(1-\tau) \%$ confidence level is defined as the $\tau$ th quantile of $Y_{t+1}$ conditional on $\mathcal{F}_{t}$, that is,

$$
\operatorname{VaR}_{t+1}(\tau)=Q_{\tau}\left(Y_{t+1} \mid \mathcal{F}_{t}\right):=\inf \left\{x: P\left(Y_{t+1} \leq x \mid \mathcal{F}_{t}\right) \geq \tau\right\}
$$

Recall that quantiles enjoy an equivariance property to monotone transformations. If $h: \mathbb{R} \rightarrow$ $\mathbb{R}$ is a nondecreasing function, $Q_{\tau}(h(Y))=h\left(Q_{\tau}(Y)\right)$ for $0<\tau<1$ and any random variable $Y$. Thus, it follows from simple algebra that the $\tau$ th conditional quantile of the price of the next day, $P_{t+1}$, is given by $Q_{\tau}\left(P_{t+1} \mid \mathcal{F}_{t}\right)=P_{t} \cdot \exp \left(Q_{\tau}\left(Y_{t+1} \mid \mathcal{F}_{t}\right) / 100\right)$. Then, the potential loss for holding the portfolio during one day is less than $P_{t}\left(1-\exp \left(Q_{\tau}\left(Y_{t+1} \mid \mathcal{F}_{t}\right) / 100\right)\right) \approx$ $P_{t} \cdot\left(-Q_{\tau}\left(Y_{t+1} \mid \mathcal{F}_{t}\right) / 100\right)$ with probability $1-\tau$.

The rest of paper is organized as follows. In Section 2, we review and extend the CAViaR models of Engle and Manganelli (2004). In Section 3, we propose the VaR forecasting using the information criteria for the quantile regression. In Section 4, an empirical study is provided to examine the performance of the proposed method. In Section 5, concluding remarks are provided. 


\section{A family of CAViaR models}

\subsection{CAViaR models}

The class of CAViaR models, proposed by Engle and Manganelli (2004), is parametric specification for the conditional quantile of asset returns and the specification is estimated via quantile regression method. Let $f_{t}(\beta)=Q_{\tau}\left(Y_{t} \mid \mathcal{F}_{t-1}\right)$ be a given parametric form for the $\tau$ th quantile of a current return $Y_{t}$ conditional on past observations. The $\tau$ th conditional quantile function is naturally estimated by minimizing the quantile regression loss function:

$$
\hat{\boldsymbol{\beta}}_{T}(\tau)=\underset{\boldsymbol{\beta}}{\operatorname{argmin}} \frac{1}{T} \sum_{t=1}^{T} \rho_{\tau}\left(Y_{t}-f_{t}(\boldsymbol{\beta})\right),
$$

where $\rho_{\tau}(u)=u(\tau-I(u<0))$ and $I(\cdot)$ is the indicator function. Note that $f_{t}(\boldsymbol{\beta})$ is a function of observations up to time $t-1$ and a parameter vector $\beta$. Given daily observations $\left\{Y_{1}, Y_{2}, \ldots, Y_{T}\right\}$, the one-day-ahead VaR forecast at $100(1-\tau) \%$ condifidence level is given by $f_{T+1}\left(\hat{\boldsymbol{\beta}}_{T}(\tau)\right)$.

An example of CAViaR models is the following symmetric absolute value specification:

$$
f_{t}(\beta)=\beta_{1}+\beta_{2} f_{t-1}(\beta)+\beta_{3}\left|Y_{t-1}\right|,
$$

where the conditional quantile has autoregressive representation. The idea of Engle and Manganelli (2004) is that the conditional quantile is tightly linked to the conditional volatility, which admits autoregressive specification in GARCH-type models, so the conditional quantile must exhibit similar behavior. In particular, $f_{t}(\boldsymbol{\beta})$ in $(2.2)$ can be deduced from the following linear $\operatorname{GARCH}(1,1)$ model introduced by Taylor (1986):

$$
Y_{t}=\sigma_{t} \eta_{t}, \quad \sigma_{t}=\gamma_{1}+\gamma_{2} \sigma_{t-1}+\gamma_{3}\left|Y_{t-1}\right|,
$$

where $\left\{\eta_{t}\right\}$ are i.i.d. random variables with the zero mean and unit variance and $\eta_{t}$ is independent of $\mathcal{F}_{t-1}$. If $\left\{Y_{t}\right\}$ follows the model (2.3), then

$$
Q_{\tau}\left(Y_{t} \mid \mathcal{F}_{t-1}\right)=Q_{\tau}\left(\eta_{t}\right) \sigma_{t}=\gamma_{1} Q_{\tau}\left(\eta_{1}\right)+\gamma_{2} Q_{\tau}\left(Y_{t-1} \mid \mathcal{F}_{t-2}\right)+\gamma_{3} Q_{\tau}\left(\eta_{1}\right)\left|Y_{t-1}\right|
$$

The conditional quantile has the form of (2.2) and the autoregressive parameter $\beta_{2}=\gamma_{2}$ remains the same.

Similarly, the asymmetric slope and indirect GARCH CAViaR specifications of Engle and Manganelli (2004) can be checked to correspond to the linear threshold-GARCH (TGARCH) introduced by Zakoïan (1994) and the standard GARCH model, respectively. Thus, all the CAViaR specifications in Engle and Manganelli (2004) are related to pure-volatility models. However, the return series of financial assets often have the non-negligible conditional mean. To incorporate this property, we design extensions of CAViaR specifications for models with a constant location parameter in the next subsection.

\subsection{Extended CAViaR models}

Suppose that $\left\{Y_{t}\right\}$ follows the equation:

$$
Y_{t}=\mu+Y_{t}^{*}=\mu+\sigma_{t} \eta_{t},
$$


where $\left\{Y_{t}^{*}\right\}$ follows a pure-volatility process and $\left\{\eta_{t}\right\}$ is the same as in (2.3). Further, if $\left\{Y_{t}^{*}\right\}$ follows the linear GARCH model (2.3), we have that

$$
Q_{\tau}\left(Y_{t}^{*} \mid \mathcal{F}_{t-1}\right)=\beta_{1}+\beta_{2} Q_{\tau}\left(Y_{t-1}^{*} \mid \mathcal{F}_{t-2}\right)+\beta_{3}\left|Y_{t-1}^{*}\right|,
$$

as given in (2.4). By using the fact that $Q_{\tau}\left(Y_{t}^{*} \mid \mathcal{F}_{t-1}\right)=Q_{\tau}\left(Y_{t} \mid \mathcal{F}_{t-1}\right)-\mu$, the CAViaR specifition $f_{t}(\boldsymbol{\beta})$ for the model $(2.5)$ is given by

$$
f_{t}(\boldsymbol{\beta})=\mu\left(1-\beta_{2}\right)+\beta_{1}+\beta_{2} f_{t-1}(\beta)+\beta_{3}\left|Y_{t-1}-\mu\right|,
$$

where $\beta=\left(\mu, \beta_{1}, \beta_{2}, \beta_{3}\right)$. In a similar way, other CAViaR specifications also can be extended to include a constant location parameter.

It is a stylized fact that past positive and negative returns have different effects on the current volatility. To capture this threshold effect, Glosten et al. (1993) proposed an extension of the standard GARCH model, which is referred to as the GJR-GARCH model. Since Engle and Manganelli (2004) considered the threshold effect only in the linear GARCH model, we examine the CAViaR specification for the GJR-GARCH model additionally.

As a consequence, we analyze $8 \mathrm{CAViaR}$ specifications presented below, which are corresponding to the standard and linear $\operatorname{GARCH}(1,1)$ models and their variants with a constant location parameter and/or the threshold effect. Recall that the symmetric absolute value, asymmetric slope, indirect GARCH specifications of Engle and Manganelli (2004) are deduced from the linear GARCH, linear TGARCH, and standard GARCH models, respectively. Below, they are referred to as CAViaR 5, 6, and 1, respectively. In the indirect GARCH CAViaR specification below, the $\tau$ th conditional quantile is set to be negative in advance since the value of $\tau$ is usually 0.01 or 0.05 . In what follows, we denote by $a^{+}=\max \{a, 0\}$ and $a^{-}=\max \{-a, 0\}$.

CAViaR 1 (standard GARCH): $\quad f_{t}(\boldsymbol{\beta})=-\left(\beta_{1}+\beta_{2} f_{t-1}^{2}(\boldsymbol{\beta})+\beta_{3} Y_{t-1}^{2}\right)^{1 / 2}$.

CAViaR 2 (GJR-GARCH): $\quad f_{t}(\boldsymbol{\beta})=-\left(\beta_{1}+\beta_{2} f_{t-1}^{2}(\boldsymbol{\beta})+\beta_{3}\left(Y_{t-1}^{+}\right)^{2}+\beta_{4}\left(Y_{t-1}^{-}\right)^{2}\right)^{1 / 2}$.

CAViaR 3 (standard GARCH with $\mu$ ):

$$
f_{t}(\boldsymbol{\beta})=\mu-\left(\beta_{1}+\beta_{2}\left(f_{t-1}(\boldsymbol{\beta})-\mu\right)^{2}+\beta_{3}\left(Y_{t-1}-\mu\right)^{2}\right)^{1 / 2} .
$$

CAViaR 4 (GJR-GARCH with $\mu)$ :

$$
f_{t}(\boldsymbol{\beta})=\mu-\left(\beta_{1}+\beta_{2}\left(f_{t-1}(\boldsymbol{\beta})-\mu\right)^{2}+\beta_{3}\left\{\left(Y_{t-1}-\mu\right)^{+}\right\}^{2}+\beta_{4}\left\{\left(Y_{t-1}-\mu\right)^{-}\right\}^{2}\right)^{1 / 2} .
$$

CAViaR 5 (linear GARCH): $\quad f_{t}(\boldsymbol{\beta})=\beta_{1}+\beta_{2} f_{t-1}(\boldsymbol{\beta})+\beta_{3}\left|Y_{t-1}\right|$.

CAViaR 6 (linear TGARCH): $\quad f_{t}(\boldsymbol{\beta})=\beta_{1}+\beta_{2} f_{t-1}(\boldsymbol{\beta})+\beta_{3} Y_{t-1}^{+}+\beta_{4} Y_{t-1}^{-}$.

CAViaR 7 (linear GARCH with $\mu$ ):

$$
f_{t}(\boldsymbol{\beta})=\mu\left(1-\beta_{2}\right)+\beta_{1}+\beta_{2} f_{t-1}(\boldsymbol{\beta})+\beta_{3}\left|Y_{t-1}-\mu\right| .
$$

CAViaR 8 (linear TGARCH with $\mu$ ):

$$
f_{t}(\boldsymbol{\beta})=\mu\left(1-\beta_{2}\right)+\beta_{1}+\beta_{2} f_{t-1}(\boldsymbol{\beta})+\beta_{3}\left(Y_{t-1}-\mu\right)^{+}+\beta_{4}\left(Y_{t-1}-\mu\right)^{-} .
$$




\section{VaR forecasting using information criteria}

\subsection{Encompassing CAViaR models}

It is a practical problem to select a CAViaR model among several specifications. One simple and fast way is to utilize the Akaike and Bayesian information criteria. Since the CAViaR method is based on quantile regression, the AIC or BIC for quantile regression is well-suited for the selection of CAViaR models.

Hurvich and Tsai (1990) considered the AIC for median regression. They interpreted the absolute deviation estimator as the maximum likelihood estimator (MLE) when errors follow a double exponential distribution. Machado (1993) studied the BIC for general $M$-estimation including the median regression. Recently, Behl et al. (2013) proposed a focused information criterion for the quantile regression.

For the quantile regression problem (2.1), the AIC and BIC of the $j$ th model are given as

$$
A I C_{j}=2 T \log \hat{\sigma}_{j}+2 p_{j}, \quad B I C_{j}=2 T \log \hat{\sigma}_{j}+p_{j} \log T
$$

where $\hat{\sigma}_{j}=T^{-1} \sum_{t=1}^{T} \rho_{\tau}\left(Y_{t}-f_{t}\left(\hat{\boldsymbol{\beta}}_{j}(\tau)\right)\right.$ and $p_{j}$ denotes the dimension of the parameter space containing $\beta_{j}$, the parameter vector of the $j$ th model: see, for example, Behl et al. (2013) and Koenker (2005).

One explanation for (3.1) can be provided as follows. As a underlying model for the quantile regression, consider the following model given in Engle and Manganelli (2004):

$$
Y_{t}=f_{t}(\beta)+\varepsilon_{\tau t}
$$

where $f_{t}(\boldsymbol{\beta})$ is a function of $\left\{Y_{t-1}, Y_{t-2}, \ldots\right\}$ and $Q_{\tau}\left(\varepsilon_{\tau t} \mid \mathcal{F}_{t-1}\right)=0$. According to Koenker and Machado (1999) and Yu and Moyeed (2001), the quantile regression estimator $\hat{\boldsymbol{\beta}}(\tau)$ can be interpreted as a quasi-MLE assuming that $\varepsilon_{\tau t}$ follows an asymmetric Laplace distribution having the density function:

$$
f_{\tau}(u ; b)=\frac{\tau(1-\tau)}{b} \exp \left\{\frac{-\rho_{\tau}(u)}{b}\right\}, \quad b>0 .
$$

Then, the AIC in (3.1) is obtained by ignoring an additive constant $-2 T \log \tau(1-\tau)+2 T+2$.

Now, we propose a simple method for the VaR forecasting in which several CAViaR models are encompassed by using the AIC or BIC for quantile regression. This method takes into account the possibility that the CAViaR specification may change over time. At each time, we select a CAViaR model minimizing the information criterion given in (3.1) among candidate models. Hence, we update not only the model parameters but also the model specifications.

Since the selection procedure needs only minimized values of the quantile regression loss functions, no additional burden of calculation is required. But the performance of the proposed VaR forecasts is quite reasonable and more stable than those by any single CAViaR model as will be shown in Section 4 below.

\subsection{Assessing the quality of VaR forecasts}

In the VaR literature, various backtesting measures have been proposed to assess the quality of VaR forecasts. Denote by $\widehat{\operatorname{VaR}}_{t}(\tau)$ the one-step-ahead VaR forecast at $100(1-\tau) \%$ 
confidence level evaluated at time $t-1$. Let $t \in\{1,2, \ldots, N\}$ be the forecasting period and $I_{t}=I\left(Y_{t}<\widehat{\operatorname{VaR}}_{t}(\tau)\right)$ be the indicator of VaR violation. As pointed out in Christoffersen (1998), the definition of $\operatorname{VaR}_{t}(\tau)$ in (1.1) implies that the true VaR violations, $I\left(Y_{t}<\right.$ $\operatorname{VaR}_{t}(\tau)$ ), are i.i.d. Bernoulli $(\tau)$ random variables. Hence, backtesting measures mainly test the null hypothesis that $\left\{I_{t}: 1 \leq t \leq N\right\}$ are i.i.d. Bernoulli $(\tau)$.

In this paper, we employ the following three backtesting measures among others: the unconditional coverage (UC), conditional coverage (CC), and dynamic quantile (DQ) tests of Kupiec (1995), Christoffersen (1998) and Engle and Manganelli (2004), respectively. The $\mathrm{UC}$ test is a likelihood ratio (LR) test when the alternative hypothesis is that $E\left[I_{t}\right] \neq \tau$. The CC test is also a LR test against the alternative that $\left\{I_{t}\right\}$ is a first order Markov chain. The DQ test is derived from regressing $I_{t}$ on its own lagged variables and lagged VaR forecasts. For details of the backtesting, we refer to Kuester et al. (2006) and Berkowitz et al. (2011). This paper employ a variant of the DQ test, suggested by Berkowitz et al. (2011). Consider the following logistic autoregressive model:

$$
P\left[I_{t}=1 \mid \mathcal{F}_{t-1}\right]=f\left(\alpha+\sum_{j=1}^{p} \beta_{1 j} I_{t-j}+\sum_{i=1}^{q} \beta_{2 i} \widehat{\operatorname{VaR}}_{t+1-i}(\tau)\right)=: p_{t}(\boldsymbol{\theta}),
$$

where $f(x)=(1+\exp (-x))^{-1}$ and $\boldsymbol{\theta}=\left(\alpha, \beta_{11}, \ldots, \beta_{1 p}, \beta_{21}, \ldots, \beta_{2 q}\right)$. For testing the hypotheses:

$$
\begin{aligned}
& H_{0}: \alpha=\log \tau(1-\tau)^{-1}, \beta_{11}=\cdots=\beta_{1 p}=0, \beta_{21}=\cdots=\beta_{2 q}=0 \\
& H_{1}: \operatorname{not} H_{0}
\end{aligned}
$$

the LR test statistic is given by

$$
L R_{D Q}=-2 \log \left[\frac{(1-\tau)^{N-\sum_{t=1}^{N} I_{t}} \tau^{\sum_{t=1}^{N} I_{t}}}{\prod_{t=1}^{N}\left(1-p_{t}(\hat{\boldsymbol{\theta}})\right)^{1-I_{t}} p_{t}(\hat{\boldsymbol{\theta}})^{I_{t}}}\right],
$$

which asymptotically follows a chi-square distribution with $(p+q+1)$ degrees of freedom. In Section 4 , we use the above test with $p=2$ and $q=1$. The implementation would be easy by using a statistical package supporting the generalized linear models.

Notice that the above backtesting measures are designed mainly to test whether given VaR forecasts are reasonable or not. Thus, the measures can be regarded as qualitative tools for the assessment. Since we need to compare several methods for the VaR forecasting, a quantitative measure is also considered. An empirical forecasting risk can be given by

$$
\operatorname{Risk}_{\mathrm{e}}=N^{-1} \sum_{t=1}^{N} \rho_{\tau}\left(Y_{t}-\widehat{\operatorname{VaR}}_{t}(\tau)\right)
$$

For in-sample cases, the minimized value of the quantile regression loss function has been utilized to quantify goodness of fit as in Koenker and Machado (1999) and Section 3.1. The above empirical risk is its out-of-sample version. 


\section{Empirical results}

\subsection{Computational aspects}

The VaR forecasts are obtained by estimating parameters in CAViaR specifications via quantile regression. In this subsection, we address some computational details.

Consider the quantile regression problem of (2.1) with the CAViaR 8 specification in Section 2.2, for example. Recall that the specification is derived from the linear TGARCH $(1,1)$ model with a constant location parameter. Denote by $f_{t}^{*}(\boldsymbol{\beta})=f_{t}(\boldsymbol{\beta})-\mu$. To compute $f_{t}(\boldsymbol{\beta})$, we initialize $\left(Y_{0}-\mu\right)^{+},\left(Y_{0}-\mu\right)^{-}$, and $f_{0}^{*}(\beta)$ to $T^{-1} \sum_{t=1}^{T}\left(Y_{t}-\mu\right)^{+}, T^{-1} \sum_{t=1}^{T}\left(Y_{t}-\mu\right)^{-}$, and $\left(1-\beta_{2}\right)^{-1}\left\{\beta_{1}+\beta_{3} T^{-1} \sum_{t=1}^{T}\left(Y_{t}-\mu\right)^{+}+\beta_{4} T^{-1} \sum_{t=1}^{T}\left(Y_{t}-\mu\right)^{-}\right\}$, respectively. Notice that the initial values are reasonable under a stationarity assumption.

Table 4.1 VaR forecasting results for KRW/USD

\begin{tabular}{|c|c|c|c|c|c|c|c|c|c|c|}
\hline \multirow[b]{2}{*}{ Model } & \multicolumn{5}{|c|}{$95 \% \mathrm{VaR}$} & \multicolumn{5}{|c|}{$99 \% \mathrm{VaR}$} \\
\hline & $\hat{p}$ & $\mathrm{UC}$ & $\mathrm{CC}$ & $\mathrm{DQ}$ & Risk $_{\mathrm{e}}$ & $\hat{p}$ & $\mathrm{UC}$ & $\mathrm{CC}$ & $\mathrm{DQ}$ & Risk $_{\mathrm{e}}$ \\
\hline CAViaR 1 & 0.045 & 0.494 & 0.665 & 0.247 & 0.048 & 0.004 & $0.048^{*}$ & 0.139 & $0.011^{*}$ & 0.012 \\
\hline CAViaR 2 & 0.041 & 0.230 & 0.393 & 0.061 & 0.049 & 0.006 & 0.278 & 0.534 & $0.005^{*}$ & 0.014 \\
\hline CAViaR 3 & 0.042 & 0.304 & 0.528 & 0.221 & 0.048 & 0.003 & $0.013^{*}$ & $0.044^{*}$ & $0.000^{*}$ & 0.012 \\
\hline CAViaR 4 & 0.046 & 0.606 & 0.708 & 0.232 & 0.048 & 0.009 & 0.764 & 0.889 & 0.626 & 0.013 \\
\hline CAViaR 5 & 0.046 & 0.606 & 0.708 & 0.183 & 0.049 & 0.012 & 0.679 & 0.817 & $0.000 *$ & 0.014 \\
\hline CAViaR 6 & 0.046 & 0.606 & 0.806 & 0.061 & 0.049 & 0.012 & 0.679 & 0.817 & $0.000 *$ & 0.014 \\
\hline CAViaR 7 & 0.046 & 0.606 & 0.147 & $0.046^{*}$ & 0.049 & 0.013 & 0.453 & 0.654 & $0.000 *$ & 0.014 \\
\hline CAViaR 8 & 0.042 & 0.304 & 0.528 & 0.436 & 0.049 & 0.009 & 0.764 & 0.889 & 0.135 & 0.014 \\
\hline $\mathrm{CAViaR}_{\mathrm{AIC}}$ & 0.045 & 0.494 & 0.665 & 0.501 & 0.048 & 0.010 & 0.949 & 0.909 & 0.629 & 0.013 \\
\hline $\mathrm{CAViaR}_{\mathrm{BIC}}$ & 0.043 & 0.393 & 0.603 & 0.471 & 0.048 & 0.010 & 0.949 & 0.909 & 0.621 & 0.013 \\
\hline
\end{tabular}

Table 4.2 Relative frequencies of the selected CAViaR specifications for KRW/USD

\begin{tabular}{|c|c|c|c|c|c|c|c|c|}
\hline CAViaR model & 1 & 2 & 3 & 4 & 5 & 6 & 7 & 8 \\
\hline & \multicolumn{8}{|c|}{$95 \% \mathrm{VaR}$} \\
\hline $\mathrm{AIC}$ & 0.04 & 0.10 & 0.00 & 0.09 & 0.00 & 0.00 & 0.01 & 0.76 \\
\hline $\mathrm{BIC}$ & 0.10 & 0.14 & 0.00 & 0.02 & 0.01 & 0.00 & 0.00 & 0.73 \\
\hline & \multicolumn{8}{|c|}{$99 \% \mathrm{VaR}$} \\
\hline $\mathrm{AIC}$ & 0.00 & 0.00 & 0.01 & 0.52 & 0.00 & 0.00 & 0.00 & 0.47 \\
\hline $\mathrm{BIC}$ & 0.04 & 0.01 & 0.00 & 0.48 & 0.00 & 0.00 & 0.00 & 0.47 \\
\hline
\end{tabular}

NOTE: Values in bold indicate the largest frequencies.

To solve (2.1), we utilize the R function constrOptim using the Nelder-Mead algorithm. As initial values for the optimization procedure, we use the Gaussian quasi-MLE after reparameterization for which one can utilize the rugarch package and the relation between CAViaR specifications and GARCH-type models such as (2.4). A constraint of $0 \leq \beta_{2}<1$ is necessary for all the $8 \mathrm{CAViaR}$ specifications in Section 2.2. Further, constraints of nonnegativity of $\beta_{1}, \beta_{3}, \beta_{4}$ are required for CAViaR 1-4 specifications, which are variants of the standard GARCH model. 


\subsection{Real data analysis}

In this subsection, we examine and compare the VaR forecasting performance of each of 8 CAViaR models in Section 2 and the proposed methods using the AIC and BIC. We analyze the Korean won/US dollar (KRW/USD) exchange rates, the Korea Composite Stock Price Index (KOSPI), and Korean Securities Dealers Automated Quotation (KOSDAQ) Index, which are obtained from Datastream. The daily log returns are computed as 100 times the difference of the log of the prices. The returns range from August 31, 2006 to June 28, 2013.

The one-day-ahead VaR forecasting is implemented in the rolling scheme, that is, $\widehat{\operatorname{VaR}}_{t}(\tau)$ is calculated at each time $t-1$ from the last $w$ observations of daily returns. We set the size of moving windows, $w=1,000$. In fact, we also implemented the forecasting with the moving window size of $w=500$. Since the results are similar to those with $w=1,000$, we only report the case of $w=1,000$. The forecasting period is 3 years from July 1, 2010 to June 28, 2013 amounting to 782 trading days. As mentioned in Section 3.2, the performance of the $10 \mathrm{VaR}$ forecasting methods are assessed in terms of the three backtestings and the empirical forecasting risk for each of the returns and at $\tau \in\{0.01,0.05\}$.

Table 4.3 VaR forecasting results for KOSPI

\begin{tabular}{|c|c|c|c|c|c|c|c|c|c|c|}
\hline \multirow[b]{2}{*}{ Model } & \multicolumn{5}{|c|}{$95 \% \mathrm{VaR}$} & \multicolumn{5}{|c|}{$99 \% \mathrm{VaR}$} \\
\hline & $\hat{p}$ & $\mathrm{UC}$ & $\mathrm{CC}$ & DQ & Risk $_{\mathrm{e}}$ & $\hat{p}$ & $\mathrm{UC}$ & $\mathrm{CC}$ & DQ & Risk $_{\mathrm{e}}$ \\
\hline CAViaR 1 & 0.037 & 0.083 & 0.152 & 0.191 & 0.130 & 0.008 & 0.495 & 0.751 & 0.276 & 0.034 \\
\hline CAViaR 2 & 0.041 & 0.230 & 0.446 & 0.514 & 0.126 & 0.006 & 0.278 & 0.534 & 0.159 & 0.035 \\
\hline CAViaR 3 & 0.036 & 0.056 & 0.154 & 0.126 & 0.126 & 0.005 & 0.130 & 0.309 & 0.612 & 0.034 \\
\hline CAViaR 4 & 0.035 & $0.036^{*}$ & 0.107 & 0.222 & 0.126 & 0.008 & 0.495 & 0.751 & 0.516 & 0.032 \\
\hline CAViaR 5 & 0.036 & 0.056 & 0.054 & 0.157 & 0.131 & 0.008 & 0.495 & 0.751 & 0.276 & 0.035 \\
\hline CAViaR 6 & 0.038 & 0.120 & 0.284 & 0.321 & 0.126 & 0.005 & 0.130 & 0.309 & 0.255 & 0.038 \\
\hline CAViaR 7 & 0.037 & 0.083 & 0.214 & 0.226 & 0.129 & 0.006 & 0.278 & 0.534 & 0.863 & 0.037 \\
\hline CAViaR 8 & 0.036 & 0.056 & 0.154 & 0.349 & 0.128 & 0.005 & 0.130 & 0.309 & 0.467 & 0.036 \\
\hline $\mathrm{CAViaR}_{\mathrm{AIC}}$ & 0.037 & 0.083 & 0.214 & 0.456 & 0.127 & 0.006 & 0.278 & 0.534 & 0.744 & 0.034 \\
\hline $\mathrm{CAViaR}_{\mathrm{BIC}}$ & 0.037 & 0.083 & 0.214 & 0.455 & 0.128 & 0.006 & 0.278 & 0.534 & 0.729 & 0.034 \\
\hline
\end{tabular}

NOTES: Values in bold indicate the largest $p$-values and the smallest empirical forecasting risk among 10 methods. The symbol $*$ denotes rejection at $5 \%$ significance level.

Table 4.4 Relative frequencies of the selected CAViaR specifications for KOSPI

\begin{tabular}{|c|c|c|c|c|c|c|c|c|}
\hline CAViaR model & $\overline{1}$ & 2 & 3 & $\overline{4}$ & $\overline{5}$ & $\overline{6}$ & $\overline{7}$ & $\overline{8}$ \\
\hline & \multicolumn{8}{|c|}{$95 \% \mathrm{VaR}$} \\
\hline $\mathrm{AIC}$ & 0.00 & 0.00 & 0.01 & 0.10 & 0.00 & 0.04 & 0.41 & 0.44 \\
\hline $\mathrm{BIC}$ & 0.00 & 0.00 & 0.02 & 0.08 & 0.00 & 0.07 & 0.65 & 0.17 \\
\hline \multicolumn{9}{|c|}{$99 \% \mathrm{VaR}$} \\
\hline $\mathrm{AIC}$ & 0.00 & 0.02 & 0.00 & 0.27 & 0.00 & 0.00 & 0.11 & 0.60 \\
\hline $\mathrm{BIC}$ & 0.00 & 0.02 & 0.01 & 0.26 & 0.00 & 0.00 & 0.19 & 0.52 \\
\hline
\end{tabular}

NOTE: Values in bold indicate the largest frequencies.

Tables 4.1, 4.3 and 4.5 report the forecasting results for KRW/USD, KOSPI and KOSDAQ, respectively. The tables present the coverage rates $(\hat{p})$, which are the observed proportions of VaR violations, $p$-values of the UC, CC and DQ tests, and the empirical forecasting risk in (3.2). Since a large $p$-value indicates good performance indirectly, the largest $p$-values in each test are denoted in bold in the tables. Tables 4.2, 4.4 and 4.6 show the selection frequencies by the AIC and BIC in each series. First of all, the results reveal that the two information 
criteria based methods pass the backtestings at $5 \%$ significance level in all cases. Thus, our proposed methods show reasonable performance, though the methods do not outperform others all the time. Each of 8 CAViaR models also pass the most backtestings but except the case of $99 \%$ VaR for KRW/USD.

Table 4.5 VaR forecasting results for KOSDAQ

\begin{tabular}{|c|c|c|c|c|c|c|c|c|c|c|}
\hline \multirow[b]{2}{*}{ Model } & \multicolumn{5}{|c|}{$95 \% \mathrm{VaR}$} & \multicolumn{5}{|c|}{$99 \%$ VaR } \\
\hline & $\hat{p}$ & $\mathrm{UC}$ & $\mathrm{CC}$ & $\mathrm{DQ}$ & Risk $_{\mathrm{e}}$ & $\hat{p}$ & $\mathrm{UC}$ & $\mathrm{CC}$ & $\mathrm{DQ}$ & Risk $_{e}$ \\
\hline CAViaR 1 & 0.049 & 0.856 & 0.930 & 0.944 & 0.165 & 0.009 & 0.764 & 0.889 & 0.891 & 0.045 \\
\hline CAViaR 2 & 0.045 & 0.494 & 0.424 & 0.585 & 0.164 & 0.009 & 0.764 & 0.889 & 0.893 & 0.045 \\
\hline CAViaR 3 & 0.043 & 0.393 & 0.606 & 0.887 & 0.160 & 0.010 & 0.949 & 0.909 & 0.931 & 0.044 \\
\hline CAViaR 4 & 0.041 & 0.230 & 0.393 & 0.682 & 0.160 & 0.010 & 0.949 & 0.181 & 0.394 & 0.044 \\
\hline CAViaR 5 & 0.045 & 0.494 & 0.424 & 0.762 & 0.167 & 0.010 & 0.949 & 0.181 & 0.340 & 0.045 \\
\hline CAViaR 6 & 0.042 & 0.304 & 0.497 & 0.820 & 0.162 & 0.009 & 0.764 & 0.133 & 0.329 & 0.045 \\
\hline CAViaR 7 & 0.043 & 0.393 & 0.606 & 0.895 & 0.161 & 0.010 & 0.949 & 0.181 & 0.407 & 0.045 \\
\hline CAViaR 8 & 0.041 & 0.230 & 0.393 & 0.698 & 0.160 & 0.012 & 0.679 & 0.817 & 0.556 & 0.043 \\
\hline $\mathrm{CAViaR}_{\mathrm{AIC}}$ & 0.042 & 0.304 & 0.497 & 0.806 & 0.160 & 0.010 & 0.949 & 0.909 & 0.786 & 0.043 \\
\hline $\mathrm{CAViaR}_{\mathrm{BIC}}$ & 0.042 & 0.304 & 0.497 & 0.839 & 0.161 & 0.012 & 0.679 & 0.817 & 0.960 & 0.044 \\
\hline
\end{tabular}

NOTES: Values in bold indicate the largest $p$-values and the smallest empirical forecasting risk among 10 methods. The symbol $*$ denotes rejection at $5 \%$ significance level.

Table 4.6 Relative frequencies of the selected CAViaR specifications for KOSDAQ

\begin{tabular}{ccccccccc}
\hline \hline CAViaR model & 1 & 2 & 3 & 4 & 5 & 6 & 7 & 8 \\
\hline & & \multicolumn{7}{c}{$95 \%$ VaR } \\
AIC & 0.00 & 0.00 & 0.00 & 0.10 & 0.00 & 0.00 & 0.26 & $\mathbf{0 . 6 3}$ \\
BIC & 0.00 & 0.00 & 0.03 & 0.05 & 0.00 & 0.01 & $\mathbf{0 . 8 3}$ & 0.09 \\
\hline & \multicolumn{7}{c}{$99 \%$ VaR } \\
AIC & 0.00 & 0.00 & 0.00 & 0.01 & 0.00 & 0.00 & 0.39 & $\mathbf{0 . 6 1}$ \\
BIC & 0.00 & 0.00 & 0.00 & 0.00 & 0.00 & 0.00 & $\mathbf{0 . 5 7}$ & 0.43 \\
\hline
\end{tabular}

NOTE: Values in bold indicate the largest frequencies.

In this case, the forecasting results of the AIC and BIC based methods are remarkable. Even though the methods using a single specification show poor results in the backtestings, especially in the DQ test, the performance is quite improved by encompassing multiple specifications via the AIC and BIC. Further, Table 4.2 shows that the AIC and BIC selected the better models, which are the CAViaR 4 and 8 in this case. It is also noteworthy that the proposed methods outperformed each forecasting by the CAViaR 4 and 8 . Figure 4.1 illustrates $99 \%$ VaR forecasting results for KRW/USD returns by the CAViaR 3, 4 and 8 specifications and the AIC based method. In terms of backtesting measures, forecasts by CAViaR 3 are not recommended while others are reasonable. The figure shows that the forecasts by the AIC based method resemble those by CAViaR 8 in the first half period, while they are closer to those by $C A V i a R 4$ in the second half period.

Let us turn to the empirical forecasting risk. In each series and confidence level, the forecasting risk differs by a small amount. We can see that the risk of the proposed methods are ranked first or second in the most cases. As anticipated, the AIC based method shows slightly better forecasting risk than the BIC based method. 
(a)

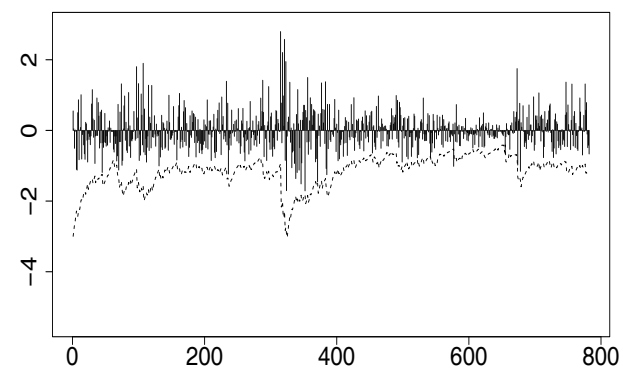

(c)

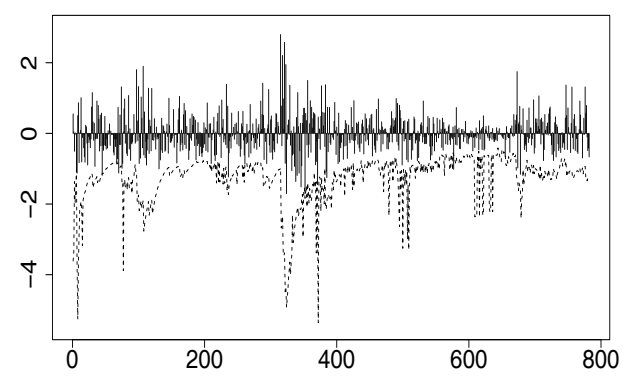

(b)

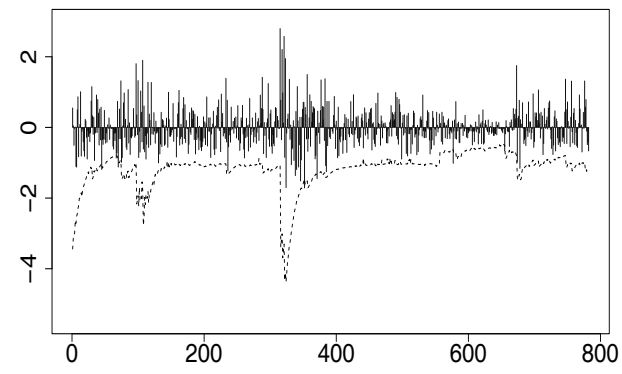

(d)

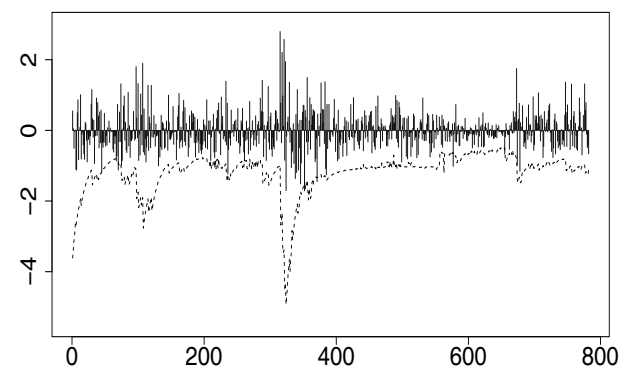

Figure 4.1 99\% VaR forecasts for KRW/USD returns using (a) CAViaR 3 (b) CAViaR 4 (c) CAViaR 8 (d) CAViaR $_{\text {AIC }}$

\section{Concluding remarks}

This paper proposed an extension of the CAViaR method of Engle and Manganelli (2004) using information criteria for quantile regression. In the new VaR forecasting, we encompass a family of CAViaR models using the AIC or BIC, thus we admit time-varying CAViaR specifications. An empirical study indicates that this method shows more stable performance than the method using a single specification.

On the other hand, this study also exposes new tasks regarding the selection problem for VaR models. Below, we address two issues which our method still can not overcome. First, it is also an important factor how many past observations would be used for the estimation, that is, the selection problem of the size of moving window. As mentioned by Gargallo et al. (2010), there is a trade-off between the precision of estimation and the model goodness of fit. Since the information criteria can not handle the cases with different sample sizes, the method of this paper is not suitable for the selection of the moving window size. Second, as seen in Section 3.1, the existing AIC and BIC for quantile regression do not take the heteroscedasticity into account while the CAViaR method is related to the quantile regression for conditionally heteroscedastic time series. This suggests a need for studying information criteria for quantile regression in heteroscedastic models. We leave these issues as tasks for the future study. 


\section{References}

Bao, Y., Lee, T.-H. and Saltoğlu, B. (2006). Evaluating predictive performance of value-at-risk models in emerging markets: A reality check. Journal of Forecasting, 25, 101-128.

Behl, P., Claeskens, G. and Dette, H. (2013). Focused model selection in quantile regression. Statistica Sinica, preprint.

Berkowitz, J., Christoffersen, P. and Pelletier, D. (2011). Evaluating value-at-risk models with desk-level data. Management Science, 57, 2213-2227.

Choi, M. S., Park, J. A. and Hwang, S. Y. (2007). Multivariate GARCH and its application to bivariate time series. Journal of the Korean Data \& Information Science Society, 18, 915-925.

Christoffersen, P. F. (1998). Evaluating interval forecasts. International Economic Review, 39, 841-862.

Engle, R. F. and Manganelli, S. (2004). CAViaR: Conditional autoregressive value at risk by regression quantiles. Journal of Business 83 Economic Statistics, 22, 367-381.

Gargallo, P., Miguel, J., Olave, P. and Salvador, M. (2010). Evaluating value at risk using selection criteria of the model and the information set. Applied Financial Economics, 20, 1415-1428.

Glosten, L. R., Jagannathan, R. and Runkle, D. E. (1993). On the relation between the expected value and the volatility of the nominal excess return on stocks. The Journal of Finance, 48, 1779-1801.

Hurvich, C. M. and Tsai, C.-L. (1990). Model selection for least absolute deviations regression in small samples. Statistics \&3 Probability Letters, 9, 259-265.

Kim, E. Y. and Lee, T. W. (2011). A numerical study on portfolio VaR forecasting based on conditional copula. Journal of the Korean Data $\&$ Information Science Society, 22, 1065-1074.

Koenker, R. (2005). Quantile regression, Econometric Society Monographs, 38, Cambridge University Press, Cambridge.

Koenker, R. and Machado, J. A. F. (1999). Goodness of fit and related inference processes for quantile regression. Journal of the American Statistical Association, 94, 1296-1310.

Kuester, K., Mittnik, S. and Paolella, M. S. (2006). Value-at-risk prediction: A comparison of alternative strategies. Journal of Financial Econometrics, 4, 53-89.

Kupiec, P. H. (1995). Techniques for verifying the accuracy of risk measurement models. Journal of Derivatives, 3, 73-84.

Lee, S. and Noh, J. (2010). Value at risk forecasting based on quantile regression for GARCH models. The Korean Journal of Applied Statistics, 23, 669-681.

Machado, J. A. F. (1993). Robust model selection and $M$-estimation. Econometric Theory, 9, 478-493.

Shim, J. and Hwang, C. (2011). Forecasting volatility via conditional autoregressive value at risk model based on support vector quantile regression. Journal of the Korean Data 86 Information Science Society, 22, $589-596$.

Taylor, S. J. (1986). Modelling financial time series, Wiley, New York.

Yu, K. and Moyeed, R. A. (2001). Bayesian quantile regression. Statistics \& Probability Letters, 54, 437-447.

Zakoïan, J.-M. (1994). Threshold heteroskedastic models. Journal of Economic Dynamics and Control, 18, 931-955. 